\title{
The Fitness Fatness Index Is Inversely Associated with Measures of Vascular Aging Derived from Blood Pressure in a Representative Sample of Adults in the United States
}

\author{
Kevin Heffernan', Paul Loprinzi \\ 'Department of Exercise Science, Syracuse University, Syracuse, NY, ${ }^{2}$ Department of Health, Exercise Science and \\ Recreation Management, University of Mississippi, Oxford, MS, USA
}

Purpose: Both low cardiorespiratory fitness (CRF) and obesity may hasten vascular aging and increase atherosclerotic cardiovascular disease (ASCVD) risk. This study explored the joint association of CRF and obesity with ASCVD risk using the fitness fatness index (FFI) and measures of vascular aging derived from blood pressure in a large sample of United States adults.

Methods: Data from the 1999-2002 National Health and Nutrition Examination Survey were used $(\mathrm{N}=8,080$ adults between the ages of $20-85$ years, which is a weighted population equivalent of 174,277,151). The FFI was calculated as CRF divided by waist-to-height ratio. Measures of vascular aging included estimated pulse wave velocity (ePWV), pulse pressure (PP), and the vascular overload index (VOI), with ePWV being calculated from systolic and diastolic blood pressures and age while PP and VOI were calculated from blood pressure alone.

Results: After full adjustment for age, sex, race/ethnicity, education, body mass index, smoking, physical activity, diabetes, hypertension, cardiovascular disease, high-density lipoprotein cholesterol, and total cholesterol, FFI was inversely associated with ePWV ( $b=-.10 ; 95 \%$ confidence interval $[C l],-0.11$ to $-0.09 ; p<0.001)$, PP $(b=-.86 ; 95 \%$ $\mathrm{Cl},-1.03$ to $-0.68 ; p<0.001)$ and the VOI $(b=-.74 ; 95 \% \mathrm{Cl},-0.95$ to $-0.053 ; p<0.001)$.

Conclusion: $\mathrm{FFI}$ is associated with lower ePWV, PP, and VOI in a nationally representative sample of adults. Maintaining higher CRF concomitant with lower body fat may minimize vascular load and contribute to healthy vascular aging. Conversely, lower fitness and higher fatness may contribute to early/accelerated vascular aging.

Keywords: Vascular stiffness, Fatness fitness index, Blood pressure

\section{Introduction}

Cardiorespiratory fitness (CRF) is a powerful predictor of atherosclerotic cardiovascular disease (ASCVD) risk, with higher

Received: July 26, $2021 \quad$ Revised: August 2, 2021

Accepted: August 6, 2021

Correspondence: Kevin Heffernan

Department of Exercise Science, Syracuse University, Syracuse, NY 13244, USA

Tel: + 1-315-443-9801, Fax: + 1-315-443-9375

E-mail: ksheffer@syr.edu

Support for this study came from the Center for Aging and Policy Studies - P30AG066583-01S1 (PI Jennifer Karas Montez).

Copyright (C2021 The Korean Society of Sports Medicine

(C) This is an Open Access article distributed under the terms of the Creative Commons Attribution Non-Commercial License (http://creativecommons.org/ licenses/by-nc/4.0) which permits unrestricted non-commercial use, distribution, and reproduction in any medium, provided the original work is properly cited.
CRF being cardioprotective and lower CRF serving as a potent incendiary for cardiometabolic disease, morbidity, and mortality ${ }^{1}$. While the prevalence of low CRF is high', so too is the prevalence of obesity. Obesity levels have reached epidemic proportions, also serving to increase ASCVD risk and cardiovascular disease (CVD)- and all-cause mortality in the general population ${ }^{3}$. Thus, the consideration of both CRF and obesity together may offer more insight into ASCVD risk than either alone.

The fitness fatness index (FFI) combines measures of CRF and fatness into a single continuous metric. CRF is determined from a validated algorithm recently shown in a meta-analysis to be an independent predictor of cardiovascular- and all-cause mortality ${ }^{4}$. Fatness is assessed using the waist-to-height ratio (WHtR), overcoming many of the potential limitations of using body mass index (BMI) as a measure of fatness ${ }^{5}$. WHtR is an 
anthropometric index known to be universally appropriate across race, age, sex, and genetic predisposition to changes in body weight and CRF level'. Moreover, the WHtR may be more closely associated with cardiometabolic risk and may be a better predictor of visceral fat than other anthropometric measures such as BMI? Thus, the FFI has been shown to be a better predictor of incident diabetes than either CRF or obesity alone, ${ }^{8,9}$, with recent studies supporting the FFI as predictive of CVD-specific and all-cause mortality in adults with CVD and the general population alike ${ }^{10,11}$. Whether the FFI is sensitive to differences in subclinical ASCVD risk remains unexplored. Such an evaluation may provide insight into the explanation of the previously observed effects between FFI and CVD- and all-cause mortality.

Measures of vascular aging offer novel insight into ASCVD risk. With aging and/or the presence of disease, large (central) arteries, such as the aorta, stiffen, exposing target organs and the downstream microvasculature to potentially damaging pulsatile hemodynamic forces ${ }^{12}$. Excessive pulsatility contributes to vascular remodeling and increases in arteriolar resistance ${ }^{13}$, which augments pressure from wave reflections, leading to further increases in pressure pulsatility. The sum effect of this hemodynamic sequela (i.e., increased arterial stiffness and pulse pressure [PP]) has been referred to as vascular overload ${ }^{14}$. Increased arterial stiffness and PP are each associated with subclinical target organ damage and future CVD risk ${ }^{12}$. We and others have found that while higher CRF is associated with lower arterial stiffness ${ }^{15}$, obesity is associated with increased arterial stiffness ${ }^{16}$.

We sought to explore the joint association of CRF and body fatness with ASCVD risk. To this end, we examined the association of FFI with estimated pulse wave velocity (ePWV), PP, and the vascular overload index (VOI) as measures of vascular aging derived from brachial blood pressure (BP) that capture the overall burden of arterial stiffness and BP pulsatility on the cardiovascular system (i.e., vascular overload). We hypothesized that a higher FFI would be associated with lower ePWV, PP, and thus, a lower VOI. We tested this hypothesis in a large, representative sample of the United States adults from the 1999-2002 National Health and Nutrition Examination Survey (NHANES; N=8,080 which is a weighted population equivalent of $174,277,151$ ).

\section{Methods}

Data from the 1999-2002 NHANES were extracted. Analyses are based on complete data from 8,080 male and female (aged 20-85 years). All NHANES procedures have been ethically approved by the National Center for Health Statistics review board. Prior to any data collection, participant consent was obtained from all individuals. Additional information on NHANES methodology and data collection can be obtained from the NHANES website (http://www.cdc.gov/nchs/nhanes.htm). Further, all NHANES survey response rates have been published elsewhere (https://wwwn.cdc.gov/nchs/nhanes/ResponseRates.aspx).

\section{Fitness fatness index}

FFI was taken as the ratio of CRF to WHtR. The WHtR was assessed directly from measured height and waist circumference measured just above the ilium using standard procedures. CRF was determined from a prediction algorithm that uses non-exercise testing methods to estimate a CRF level. These algorithms, which demonstrate evidence of predictive validity, have been shown to inversely associated with all-cause and CVD-specific mortality. The specific algorithms used were as follows: estimated CRF for female $(\mathrm{METs})=14.7873+($ age $\times 0.1159)-\left(\mathrm{age}^{2} \times 0.0017\right)-(\mathrm{BMI} \times$ $0.1534)-(\mathrm{WC} \times 0.0085)-(\mathrm{RHR} \times 0.0364)+($ active $\times 0.5987)-$ (smoker $\times 0.2994)$; estimated $\mathrm{CRF}$ for male $(\mathrm{MET})=21.2870+$ $($ age $\times 0.1654)-\left(\operatorname{age}^{2} \times 0.0023\right)-(\mathrm{BMI} \times 0.2318)-(\mathrm{WC} \times 0.0337)-$ $(\mathrm{RHR} \times 0.0390)+($ active $\times 0.6351)-($ smoker $\times 0.4263)$.

In this algorithm, MET represents metabolic equivalent of task, RHR represents resting heart rate (bpm), and WC represents waist circumference $(\mathrm{cm})$. Smoking status was determined based on self-report; current smoking was coded as 1 , and otherwise, it was coded as 0 . Activity status was determined from self-report and defined as $\geq 2,000$ MVPA MET-min-month; MVPA represents moderate-to-vigorous physical activity. Those above this threshold were coded as 1 and others were coded as 0 . The BMI $\left(\mathrm{kg} / \mathrm{m}^{2}\right)$ was determined here from measured height and weight.

\section{Estimated pulse wave velocity}

ePWV was determined from the following algorithm ${ }^{17}: 9.587-$ $(0.402 \times$ age $)+\left(4.560 \times 0.001 \times\right.$ age $\left.^{2}\right)-\left(2.621 \times 0.00001 \times\right.$ age $\left.^{2} \times \mathrm{MBP}\right)+$ 
$(3.176 \times 0.001 \times$ age $\times \mathrm{MBP})-(1.832 \times 0.01 \times \mathrm{MBP})$. In this algorithm, age was expressed in years and mean BP (MBP) was calculated as $[(\mathrm{DBP})+\{0.4 \times(\mathrm{SBP}-\mathrm{DBP})\}]$, where DBP is diastolic BP and SBP is systolic BP. PP was calculated as SBP-DBP (above).

The VOI was calculated as follows ${ }^{14}: 1.33 \times \mathrm{SBP}-0.33 \times \mathrm{DBP}-$ 133.3.

BP was obtained with participants in the seated position following 5 minutes of quiet rest. NHANES technicians completed a thorough BP training program (Shared Care Research and Education Consulting) that involved a didactic section, audio-video observation, and measurement of BP against a certified, gold standard BP instructor. For the calculation of MBP, the average of up to four BP assessments was utilized.

\section{Covariates}

Covariates included various demographic, behavioral, and CVD risk factors that could confound the association between FFI and measures of vascular aging. These included age (years), sex

Table 1. Univariate characteristics of the study variables among American Participants*

\begin{tabular}{lc}
\multicolumn{1}{c}{ Variable } & Point estimate \\
\hline \hline Dependent variable & \\
ePWV (m/sec) & $8.06 \pm 0.04$ \\
Pulse pressure (mm Hg) & $50.92 \pm 0.45$ \\
Vascular overload index (mm Hg) & $6.71 \pm 0.57$ \\
Independent variable & \\
Fitness fatness index & $18.05 \pm 0.14$ \\
Age (yr) & $45.76 \pm 0.32$ \\
Female sex (n, \%) & $4,260(52.7)$ \\
Race-ethnicity, white (n, \%) & $4,044(50.1)$ \\
College education, (n, \%) & $1,540(19.1)$ \\
Physical activity (MET-min-mo) & $4,702.9 \pm 233.7$ \\
Smoking ( $\mathrm{n}, \%)$ & $1,740(21.5)$ \\
Body mass index (kg/m $\left.{ }^{2}\right)$ & $27.94 \pm 0.14$ \\
Diabetes mellitus ( $\mathrm{n}, \%)$ & $735(9.1)$ \\
Hypertension (n, \%) & $2,367(29.3)$ \\
Cardiovascular disease (n, \%) & $322(4.0)$ \\
C-reactive protein (mg/dL) & $0.42 \pm 0.01$ \\
Total cholesterol (mg/dL) & $203.52 \pm 0.90$ \\
HDL cholesterol (mg/dL) & $51.26 \pm 0.38$ \\
\hline
\end{tabular}

Values are presented as mean \pm standard error or number (\% for unweighted value).

ePWV: estimated pulse wave velocity, MET: metabolic equivalent of task, HDL: high-density lipoprotein.

$\star 8,080$ Adults (aged 20-85 years weighted population, $174,277,151)$ (male/female), race-ethnicity (Mexican American, other Hispanic, non-Hispanic white, non-Hispanic black, and multi-race), education (college graduate or not), self-reported smoking status (smoker vs. nonsmoker), self-reported physical activity (METmin-month), measured BMI $\left(\mathrm{kg} / \mathrm{m}^{2}\right)$, physician-diagnosed diabetes/ use of diabetes medication (yes/no), physician-diagnosed hypertension/ use of anti-hypertensives (yes/no), physician-diagnosed CVD, and laboratory-assessed CVD biomarkers, including high-density lipoprotein cholesterol $(\mathrm{mg} / \mathrm{dL})$, total cholesterol $(\mathrm{mg} / \mathrm{dL})$, and C-reactive protein (CRP) (mg/dL). Specific details on the methodological assessment of these covariates can be found on the NHANES website (http://www.cdc.gov/nchs/nhanes.htm).

\section{Statistical analyses}

All statistical analyses were performed via complex survey analytical procedures using Stata version 12 (SAS Institute, Cary, NC, USA). To account for oversampling, nonresponse, noncoverage, and to provide nationally representative estimates, all analyses included the use of survey sample weights, clustering and primary sampling units.

Weighted multivariable linear regression models were used to examine the association between FFI as the independent variable with ePWV, PP, and the VOI as three separate dependent variables. Three specific models were explored, including (1) unadjusted, (2) minimally adjusted for demographic covariates (age, sex, race-ethnicity, and education), and (3) fully adjusted for demographic variables along with behavioral and CVD risk factors (BMI, smoking, physical activity, diabetes, hypertension, CVD, HDL cholesterol, total cholesterol, and CRP). To specifically explore the potential moderating impact of sex on our findings, we created a cross-product term (sex*FFI) and included it, along with its main effects, in a regression; and if significant, separate sex-specific models were explored in each of three separate regression models for ePWV, PP, and VOI. For all analyses, statistical significance was established as a nominal alpha of 0.05 .

\section{Results}

Table 1 displays the characteristics of the sample. Participants, on average, were middle age (46 years), predominately white (72.7\%), and both sexes were equally represented $(51.7 \%$ of 
females). A visual depiction of the relationship between FFI and ePWV is shown in Fig. 1. After adjusting for all the independent variables noted in Table 1, FFI was inversely associated with ePWV $(b=-.10 ; 95 \%$ confidence interval $[\mathrm{CI}],-0.11$ to -0.09 ; $\mathrm{p}<0.001)$. Results were similar in an unadjusted model $(b=-.15$; $95 \% \mathrm{CI},-0.16$ to $-0.13 ; \mathrm{p}<0.001$ ), as well as a minimally adjusted model controlling for age, sex, race-ethnicity, and education $(b=$ $-.028 ; 95 \% \mathrm{CI},-0.034$ to $-0.023 ; \mathrm{p}<0.001)$. The sex*FFI interaction term was statistically significant $(p<0.001)$; therefore, we computed separate sex-specific models (fully adjusting for all covariates) for male and female. In the fully adjusted models, FFI on ePWV for male was $b=-.117(95 \% \mathrm{CI},-0.13$ to -0.10 ; $p<0.001)$; and for female, it was $b=-.175(95 \% \mathrm{CI},-0.19$ to

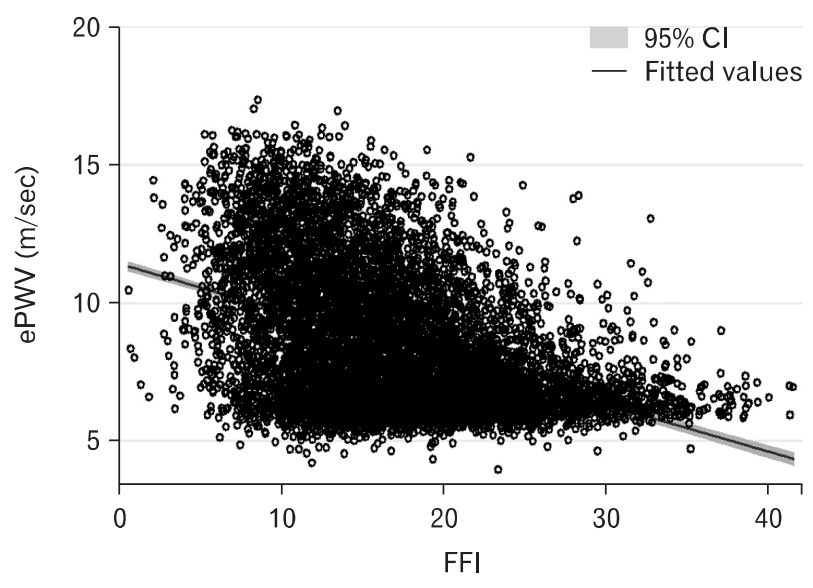

Fig. 1. Scatterplot illustrating the relationship between fitness fatness index (FFI) and estimated pulse wave velocity (ePWV) $(\mathrm{N}=8,080)$. $\mathrm{Cl}$ : confidence interval.

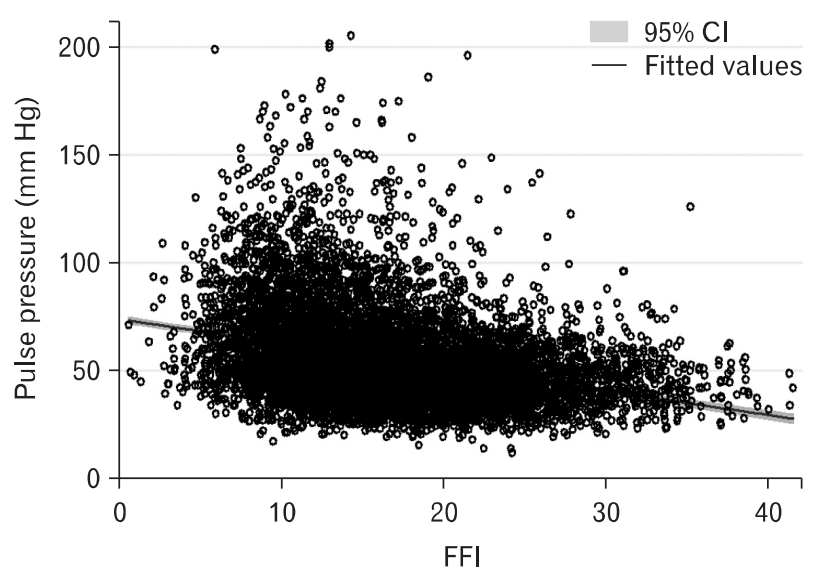

Fig. 2. Scatterplot illustrating the relationship between fitness fatness index $(\mathrm{FFI})$ and pulse pressure $(\mathrm{N}=8,080) . \mathrm{Cl}$ : confidence interval.
$-0.15 ; \mathrm{p}<0.001)$.

A visual depiction of the relationship between FFI and PP is shown in Fig. 2. After adjusting for all the independent variables noted in Table 1 , FFI was inversely associated with PP $(b=-.86$; $95 \% \mathrm{CI},-1.03$ to $-0.68 ; \mathrm{p}<0.001)$. Results were similar in an unadjusted model $(b=-.93 ; 95 \% \mathrm{CI},-1.01$ to $-0.85 ; \mathrm{p}<0.001)$, as well as a minimally adjusted model controlling for age, sex, race-ethnicity, and education $(b=-.28 ; 95 \% \mathrm{CI},-0.38$ to -0.18 ; $\mathrm{p}<0.001)$. The sex*FFI interaction term was statistically significant, $(p<0.001)$, therefore, we computed separate sex-specific models (fully adjusting for all covariates) for male and female. In the fully adjusted models, FFI on PP for male was $b=-1.10(95 \% \mathrm{CI},-1.30$ to $-0.90 ; \mathrm{p}<0.001)$; and for female, it was $b=-1.89(95 \% \mathrm{CI},-2.22$ to $-1.56 ; \mathrm{p}<0.001)$.

A visual depiction of the relationship between FFI and VOI is shown in Fig. 3. After adjusting for all the independent variables noted in Table 1 , FFI was inversely associated with VOI $(b=-.74$; $95 \% \mathrm{CI},-0.95$ to $-0.53 ; \mathrm{p}<0.001)$. Results were similar in an unadjusted model $(b=-1.26$; $95 \% \mathrm{CI},-1.35$ to -1.17 ; $<<0.001$ ), as well as a minimally adjusted model controlling for age, sex, race-ethnicity, and education $(b=-.60 ; 95 \% \mathrm{CI},-0.74$ to -0.45 ; $\mathrm{p}<0.001)$. The sex*FFI interaction term was statistically significant $(p<0.001)$; therefore, we computed separate sexspecific models (fully adjusting for all covariates) for males and females. In the fully adjusted models, FFI on the VOI for male was $b=-1.09(95 \% \mathrm{CI},-1.35$ to $-0.83 ; \mathrm{p}<0.001)$; and for female, it was $b=-1.67(95 \% \mathrm{CI},-2.07$ to $-1.25 ; \mathrm{p}<0.001)$.

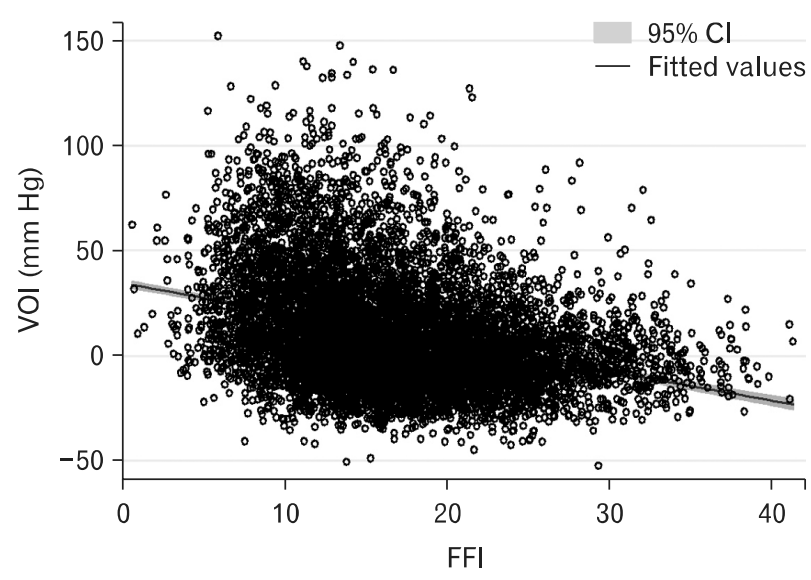

Fig. 3. Scatterplot illustrating the relationship between fitness fatness index ( $F F I)$ and vascular overload index (VOI) $(\mathrm{N}=8,080)$. Cl: confidence interval. 


\section{Discussion}

Studies that explore the interaction between CRF and obesity as related to ASCVD risk typically categorize individuals into broad fit, unfit, obese, and/or nonobese groups (e.g., fat-but-fit) using somewhat arbitrary operational definitions and cut points. For example, body fatness is typically operationalized using BMI. BMI is regularly questioned as the most parsimonious way to appraise obesity status at the population level owing to concerns around it accurately reflecting adiposity and body composition (lean mass) across age, sex, race/ethnicity, etc. ${ }^{5}$. Moreover, using such an "and/or" categorical approach may obscure more subtle intraindividual variation in fitness-fatness interactions and only offer insight into more robust shifts in risk across/between categories $^{9}$. Changes in fitness and fatness that may take place with lifestyle modification often occur concomitantly and on a continuum. A better understanding of the synergistic effects of CRF and obesity as continuous variables may be needed to inform more effective clinical practice. Thus, the FFI has been put forth as a metric to capture the joint contribution of CRF and obesity status on chronic disease risk and is emerging as a useful tool to predict cardiometabolic disease and mortality.

Our findings extend the utility of the FFI as a measure of ASCVD risk by noting associations with three separate measures of vascular aging. We found that higher FFI was independently and inversely associated with ePWV, PP, and the VOI in a large representative group of US adults from NHANES. Associations remained after adjusting for numerous covariates, including age, sex, race/ethnicity, and CVD risk factors like BMI, lipids, and CRP. Moreover, according to sex-specific analyses, while FFI was inversely associated with ePWV, PP, and VOI in both male and female, the magnitude of associations was stronger for female compared to male. These findings are taken to suggest that the combination of higher CRF and lower body fat is associated with more optimal vascular aging and by extension lower ASCVD risk.

Arterial stiffening is considered a hallmark of the vascular aging process. The gold standard method for the assessment of aortic stiffness is carotid-femoral pulse wave velocity (cfPWV) ${ }^{12}$. cfPWV can be reasonably estimated from age and mean arterial pressure $^{18}$ and this ePWV has been shown to predict CVD- and all-cause mortality independent of traditional CVD risk factors ${ }^{19}$. Age-associated reductions in arterial elasticity and loss of the intrinsic buffering capacity of large arteries owing to increased arterial stiffness contribute to an age-dependent widening of pressure pulsatility captured as PP. Elevated PP also increases ASCVD risk measured as vascular calcification ${ }^{20}$ and is an independent predictor of cardiovascular mortality. The VOI embraces both the steady (i.e. mean arterial pressure) and pulsatile (i.e., PP) components of BP and captures the effective incremental contribution of arterial stiffness, wave reflection, and arteriolar resistance to overall systolic $\mathrm{BP}^{14}$. The VOI has been shown to be associated with $\mathrm{cfPWV}^{21}$, detrimental vascular remodeling, and target organ damage (i.e. reduced brachial artery flow-mediated dilation and increased carotid intima-media thickness) ${ }^{21}$, and incident stroke ${ }^{22}$. Taken together, our findings demonstrate that FFI is independently and favorably associated with measures of vascular aging in a large sample of United States adults from NHANES.

Emerging studies note sexual dimorphism in the association of CRF and body fatness with morbidity and mortality ${ }^{23-26}$. Therefore, we explored sex-specific associations between FFI and our hemodynamic outcomes. Our findings suggest possible sex differences in the association of FFI with vascular aging. Although a higher FFI is associated with lower arterial stiffness and vascular overload in both male and female, the favorable effect of FFI on vascular aging may be stronger in female. This finding is important to note because compared to male: (1) age-associated increases in arterial stiffness and PP are greater in female; (2) the association between arterial stiffness and target organ damage, such as left ventricular hypertrophy, is greater in female; (3) the association between arterial stiffness and mortality is nearly twofold higher in female $e^{27,28}$. Thus, our findings may have important implications for sex differences in vascular aging and subsequent ASCVD risk. More research will be needed to examine optimal combinations of CRF and body fatness for preservation of vascular health in male and female.

The FFI has previously been shown to be inversely associated with $\mathrm{CRP}^{29}$, an important correlate of ASCVD. In our study, associations between FFI and vascular aging remained after adjusting for CRP, suggesting that favorable associations may be related to pathways extending beyond systemic inflammation, 
or perhaps, in the present study, CRP was not fully reflective of systemic inflammation. It is also interesting to note that associations remained after adjusting for BMI, confirming the unique contribution of WHtR to the strength of FFI in assessing ASCVD risk. Examination of the mechanisms responsible for individual contributions of high CRF, obesity status, and FFI to vascular aging are beyond the scope of this study. Higher CRF coupled with more ideal body habitus may be associated with favorable shifts in autonomic function, metabolic regulation, lipid metabolism, endothelial function, and vascular calcification; all of which may interact to favorably affect arterial stiffness and hemodynamic load ${ }^{30}$. Parenthetically, our results also support that those individuals with both low CRF, and high obesity status may be more susceptible to early vascular aging, which may drastically increase ASCVD risk.

Limitations of this study should be explored. We used an estimate of CRF derived from an established equation and not objectively measured $\mathrm{VO}_{2}$ from metabolic gas analysis. Nonetheless, estimated CRF has been shown to be a valid indirect proxy of directly measured CRF and is independently associated with CVD- and all-cause mortality ${ }^{4}$. Measures of vascular aging were all derived from systolic and diastolic BP and are likely capturing a similar hemodynamic epiphenomenon related to arterial stiffness and possibly not arterial stiffness per se. Future studies using cfPWV or brachial-ankle pulse wave velocity to measure arterial stiffness along with other established measures of vascular aging, such as carotid intima-media thickness, will be needed to confirm findings herein. The equations to estimate both FFI and ePWV included age. Although we additionally adjusted for age in our statistical models, care should be taken when interpreting our findings. To circumvent concern, we included two additional measures of vascular aging and ASCVD risk not derived from age. Associations between FFI and all three measures of vascular aging were comparable. Further, models without the control of age also produced similar findings.

In conclusion, we found that FFI was inversely associated with ePWV, PP, and the VOI in a large representative sample of the United States adults from NHANES. Maintaining higher CRF and lower body fat promotes healthy vascular aging while having both lower $\mathrm{CRF}$ and higher obesity may contribute to early vascular aging. The FFI may be a useful index to assess
ASCVD risk via its association with vascular aging in the general population.

\section{Conflict of Interest}

No potential conflict of interest relevant to this article was reported.

\section{ORCID}

Kevin Heffernan https://orcid.org/0000-0002-3499-1477

Paul Loprinzi https://orcid.org/0000-0001-7711-4741

\section{Author Contributions}

Conceptualization: KSH, PDL. Methodology: PDL. Writingoriginal draft: KSH. Writing-review \& editing: KSH, PDL.

\section{References}

1. Barry VW, Caputo JL, Kang M. The joint association of fitness and fatness on cardiovascular disease mortality: a meta-analysis. Prog Cardiovasc Dis 2018;61:136-41.

2. Carnethon MR, Gulati M, Greenland P. Prevalence and cardiovascular disease correlates of low cardiorespiratory fitness in adolescents and adults. JAMA 2005;294:2981-8.

3. Dwivedi AK, Dubey P, Cistola DP, Reddy SY. Association between obesity and cardiovascular outcomes: updated evidence from meta-analysis studies. Curr Cardiol Rep 2020;22:25.

4. Qiu S, Cai X, Sun Z, Wu T, Schumann U. Is estimated cardiorespiratory fitness an effective predictor for cardiovascular and all-cause mortality? A meta-analysis. Atherosclerosis 2021;330:22-8.

5. Donini LM, Pinto A, Giusti AM, Lenzi A, Poggiogalle E. Obesity or BMI paradox? Beneath the tip of the iceberg. Front Nutr 2020;7:53.

6. Schneider HJ, Friedrich N, Klotsche J, et al. The predictive value of different measures of obesity for incident cardiovascular events and mortality. J Clin Endocrinol Metab 2010; 95:1777-85.

7. Ashwell M, Gunn P, Gibson S. Waist-to-height ratio is a better screening tool than waist circumference and BMI for adult cardiometabolic risk factors: systematic review and meta-analysis. Obes Rev 2012;13:275-86. 
8. Sloan RA, Sawada SS, I-Min L, et al. The association of Fit-Fat Index with incident diabetes in Japanese men: a prospective cohort study. Sci Rep 2018;8:569.

9. Sloan RA, Haaland BA, Sawada SS, et al. A Fit-Fat Index for predicting incident diabetes in apparently healthy men: a prospective cohort study. PLoS One 2016;11:e0157703.

10. Edwards MK, Addoh O, Loprinzi PD. Predictive validity of a fitness fatness index in predicting cardiovascular disease and all-cause mortality. Mayo Clin Proc 2017;92:851.

11. Frith E, Loprinzi PD. The protective effects of a novel fitness-fatness index on all-cause mortality among adults with cardiovascular disease. Clin Cardiol 2017;40:469-73.

12. Townsend RR, Wilkinson IB, Schiffrin EL, et al. Recommendations for improving and standardizing vascular research on arterial stiffness: a scientific statement from the American Heart Association. Hypertension 2015;66:698-722.

13. Mitchell GF. Effects of central arterial aging on the structure and function of the peripheral vasculature: implications for end-organ damage. J Appl Physiol (1985) 2008;105:1652-60.

14. Franklin SS, Weber MA. Measuring hypertensive cardiovascular risk: the vascular overload concept. Am Heart J 1994;128:793-803.

15. Augustine JA, Yoon ES, Choo J, Heffernan KS, Jae SY. The relationship between cardiorespiratory fitness and aortic stiffness in women with central obesity. J Womens Health (Larchmt) 2016;25:680-6.

16. Kappus RM, Fahs CA, Smith D, et al. Obesity and overweight associated with increased carotid diameter and decreased arterial function in young otherwise healthy men. Am J Hypertens 2014;27:628-34.

17. Vlachopoulos C, Terentes-Printzios D, Laurent S, et al. Association of estimated pulse wave velocity with survival: a secondary analysis of SPRINT. JAMA Netw Open 2019;2: e1912831.

18. Greve SV, Blicher MK, Kruger R, et al. Estimated carotidfemoral pulse wave velocity has similar predictive value as measured carotid-femoral pulse wave velocity. J Hypertens 2016;34:1279-89.

19. Heffernan KS, Jae SY, Loprinzi PD. Association between estimated pulse wave velocity and mortality in U.S. adults. J
Am Coll Cardiol 2020;75:1862-4.

20. Heffernan KS, Barreira TV. Association between pulse pressure and aortic calcification: findings from the National Health and Nutrition Examination Survey 2013-2014. J Clin Hypertens (Greenwich) 2020;22:879-85.

21. Cai X, Xie L. A1275 relationship between vascular overload index and carotid-femoral pulse wave velocity in hypertensives. J Hypertens 2018;36:e118.

22. Yu Y, Huang Y, Liu L, et al. Association between vascular overload index and new-onset ischemic stroke in elderly population with hypertension. Clin Interv Aging 2021;16: 1293-301.

23. Al-Mallah MH, Juraschek SP, Whelton S, et al. Sex differences in cardiorespiratory fitness and all-cause mortality: the Henry Ford ExercIse Testing (FIT) Project. Mayo Clin Proc 2016;91:755-62.

24. Srikanthan P, Horwich TB, Calfon Press M, Gornbein J, Watson KE. Sex differences in the association of body composition and cardiovascular mortality. J Am Heart Assoc 2021;10:e017511.

25. Tarp J, Grøntved A, Sanchez-Lastra MA, Dalene KE, Ding D, Ekelund U. Fitness, fatness, and mortality in men and women from the UK Biobank: prospective cohort study. J Am Heart Assoc 2021;10:e019605.

26. Loprinzi PD, Cardinal BJ. Interrelationships among physical activity, depression, homocysteine, and metabolic syndrome with special considerations by sex. Prev Med 2012;54:388-92.

27. Coutinho T, Pellikka PA, Bailey KR, Turner ST, Kullo IJ. Sex differences in the associations of hemodynamic load with left ventricular hypertrophy and concentric remodeling. Am J Hypertens 2016;29:73-80.

28. Regnault V, Thomas F, Safar ME, et al. Sex difference in cardiovascular risk: role of pulse pressure amplification. J Am Coll Cardiol 2012;59:1771-7.

29. Loprinzi PD, Edwards MK. CVD-related Fit-Fat Index on inflammatory-based CVD biomarkers. Int J Cardiol 2016;223: 284-5.

30. Joyner MJ, Green DJ. Exercise protects the cardiovascular system: effects beyond traditional risk factors. J Physiol 2009; 587(Pt 23):5551-8. 